\title{
Colite colagenosa: uma causa frequente de diarreia crónica ainda subdiagnosticada
}

Sandra A. Morais, ${ }^{1}$ Rute Caçola, ${ }^{1}$ Sara Beça, ${ }^{1}$ Liliana Carneiro, ${ }^{1}$ Mrinalini Honavar, ${ }^{2}$ Rui Môço ${ }^{3}$

\section{RESUMO}

A colite microscópica é uma entidade que engloba a colite colagenosa e a colite linfocítica e é actualmente considerada uma causa comum de diarreia crónica que, apesar de benigna, tem um impacto significativo na qualidade de vida dos doentes. $O$ diagnóstico é exclusivamente histológico.

Uma vez que a colite microscópica foi descrita pela primeira vez em 1976 e apenas recentemente foi reconhecida como uma causa comum de diarreia, existem ainda muitos clínicos não sensibilizados para esta entidade.

Descrevemos o caso de um doente com diarreia crónica aquosa, inicialmente sem repercussão sistémica significativa e com exames laboratoriais e imagiológicos normais ou inconclusivos, mas que evoluiu para um quadro de choque com disfunção renal grave. Alertamos para o facto de que deverá haver uma maior insistência, por parte dos clínicos, na realização de biópsias de forma a estabelecer o correcto diagnóstico e a instituição de terapêutica adequada.

Palavras-chave: Colite Colagenosa; Colite Microscópica; Diarreia Crónica.

\section{INTRODUÇÃO}

A A colite microscópica (CM) é uma causa comum de diarreia crónica, principalmente em idosos. ${ }^{1}$ Previamente considerado um diagnóstico raro, a CM surge actualmente em 4 a $13 \%$ dos doentes estudados por diarreia crónica. ${ }^{2}$

Esta patologia engloba duas formas histológicas, a colite linfocítica e a colite colagenosa (CC), em ambas ocorrendo infiltração da lâmina própria do cólon ou íleo terminal por células inflamatórias e linfócitos intra-epiteliais, sendo que na CC se observa ainda colagéneo subepitelial $(>10 \mu \mathrm{m}) .^{3}$

A CC, a primeira forma de CM a ser descrita, ${ }^{4}$ inicialmente encarada como rara, apresenta uma incidência crescente de 0,6-5,2/100.000 indivíduos, ${ }^{2,5}$ provavelmente pelo envelhecimento da população e maior número de biópsias aquando do estudo endoscópico. ${ }^{1}$

'Médicas Internas de Medicina Interna, Serviço de Medicina Interna, Hospital Pedro Hispano, Matosinhos, Portugal.

${ }^{2}$ Médico Assistente Hospitalar de Anatomia Patológica, Serviço de Anatomia Patológica, Hospital Pedro Hispano, Portugal.

${ }_{3}^{3}$ Médico Assistente Hospitalar de Medicina Interna, Serviço de Medicina Interna, Hospital Pedro Hispano, Portugal.
Existem vários factores de risco associados ao desenvolvimento de CM, como idade avançada, género feminino, tabaco, alguns fármacos, doenças auto-imunes como a doença celíaca (DC) ou tiroidite, doenças malignas e transplante de órgão sólido., ${ }^{2,5}$

A etiologia da CM permanece ainda desconhecida, mas acredita-se que seja multifactorial, consistindo numa resposta específica da mucosa luminal a vários agentes nocivos em hospedeiro susceptível. ${ }^{5}$ Encontra-se documentada a associação com determinados fármacos, como anti-inflamatórios não-esteróides, inibidores da recaptação da serotonina, beta-bloqueadores, estatinas, bifosfonatos e inibidores da bomba de protões ${ }^{1,6}$ e agentes infecciosos como Yersinia enterocolitica, ${ }^{7}$ Clostridium difficile ${ }^{8}$ ou Campylobacter. ${ }^{9}$

Clinicamente, a CM caracteriza-se por diarreia aquosa crónica ou intermitente, não sanguinolenta. Os sintomas associados incluem manifestações digestivas como dor abdominal, tenesmo e incontinência, podendo também ocorrer manifestações sistémicas em $30 \%$ dos casos, com febrícula, astenia, perda ponderal e artralgia inflamatória. ${ }^{10}$

As manifestações laboratoriais podem incluir velo- 
cidade de sedimentação aumentada, anemia discreta, hipoalbuminemia e hipogamaglobulinemia. ${ }^{10} \mathrm{Cin}-$ quenta por cento dos doentes podem apresentar ainda positividade para factor reumatóide, anti-dsDNA, ANA's e ANCA's. ${ }^{11}$

A história natural da doença é variável. Na maioria dos casos a doença é auto-limitada, com uma duração da sintomatologia que pode variar de algumas semanas a meses. Contudo, existem situações em que a sintomatologia pode permanecer por anos num padrão contínuo ou intermitente, podendo confundir-se com doença inflamatória intestinal (DII) ou DC.

Apesar de a CM ser considerada uma doença benigna, a qualidade de vida pode encontrar-se significativamente comprometida. ${ }^{12}$

Para o diagnóstico de CM é necessária uma história clínica detalhada, dando particular ênfase à medicação crónica, factores de risco e doenças associadas. É importante o diagnóstico diferencial entre DII e DC.

Frequentemente o exame objectivo é normal e os estudos endoscópicos não revelam alterações macroscópicas. $^{5}$

O tratamento da CM deve ter em conta a severidade da sintomatologia e o impacto na qualidade de vida. A descontinuação dos factores de risco modificáveis e a promoção de alterações dietéticas, em particular a eliminação de secretagogos (cafeína e lactose) deve ser uma abordagem transversal.

Em doentes com sintomas ligeiros, muitos autores recomendam a administração de agentes antidiarreicos não específicos como a loperamida. ${ }^{1}$ Se esta terapêutica for ineficaz, ou os doentes apresentarem sintomas moderados a severos, preconiza-se o tratamento com corticóides, em particular budesonido oral $9 \mathrm{mg} / \mathrm{dia}$ durante seis a oito semanas. ${ }^{1,12-13}$ Nos doentes resistentes ou intolerantes ao budesonido, o tratamento com colestiramina, ${ }^{14}$ sulfassalazina, prednisolona $(0,5-1 \mathrm{mg} / \mathrm{kg} / \mathrm{dia})$ ou imunossupressores poderá ser considerado. Em casos refractários, em que a qualidade de vida esteja severamente comprometida, poder-se-á equacionar cirurgia ou introdução de agentes biológicos (anti-TNF). ${ }^{1}$

O curso da CM é habitualmente benigno, sem necessidade de terapêutica de manutenção. ${ }^{15}$ No entanto, pode evoluir para colite ulcerosa, DC ou doença de Crohn. ${ }^{5}$
Apresentamos o caso de um doente com quadro de diarreia crónica com repercussões clínicas graves, cujo diagnóstico definitivo só foi possível por histologia.

\section{CASO CLÍNICO}

Homem de 57 anos, reformado de trabalhador da construção civil, com hipertensão arterial, dislipidemia, antecedentes de acidente vascular cerebral sem sequelas, tabagismo activo de 50 unidades maço/ano e consumo etílico de $120 \mathrm{~g} /$ dia. Medicado com sinvastatina, lisinopril, bisoprolol, ácido acetilsalicílico, beta-histina, cloridrato de mebeverina e fermentos pancreáticos contendo lípase, pancreatina e amílase.

Inicialmente referenciado à consulta de medicina interna (MI), em 22.11.2007, por quadro com um ano de evolução de episódios intermitentes de diarreia aquosa (duas a três dejecções por dia), sem sangue, muco ou pús, mas com anorexia e perda ponderal significativa ( $13 \%$ do peso habitual). Negava outras queixas e relação com ingestão de alimentos.

O exame objectivo era normal. O estudo bioquímico revelou-se normal e não havia alterações a nível do hemograma nem aumento dos marcadores inflamatórios. Ressaltamos função tiroideia sem alterações e serologias negativas para HIV 1/2, HBV, HCV e CMV. Anticorpos anti-gliadina, anti-transglutaminase, ANA, ANCA e anti-tiroideus normais. Coprocultura (Shigella, Salmonella e Campylobacter) e exame parasitológico de fezes (Cryptosporidium parvum e Giardia lamblia) negativos.

Ecografia abdominal sem alterações de relevo e endoscopia digestiva alta (EDA) com gastrite crónica, metaplasia intestinal e pesquisa de Helicobacter pylori negativa. Endoscopia digestiva baixa com colite da transição rectosigmoideia; exame histológico (Figura 1) demonstrava preservação da arquitectura glandular, presença de infiltrado inflamatório com predomínio de polimorfonucleares, sem granulomas. Ligeiro reforço da membrana basal. Por suspeita de insuficiência pancreática exócrina foi iniciada prova terapêutica com fermentos pancreáticos e o doente foi instruído a manter abstinência alcoólica e tabágica, tendo-se verificado resolução do quadro clínico e ganho ponderal de $10 \mathrm{~kg}$.

A 22.02.2011 foi internado no serviço de MI, novamente com diarreia aquosa com as mesmas caracte- 


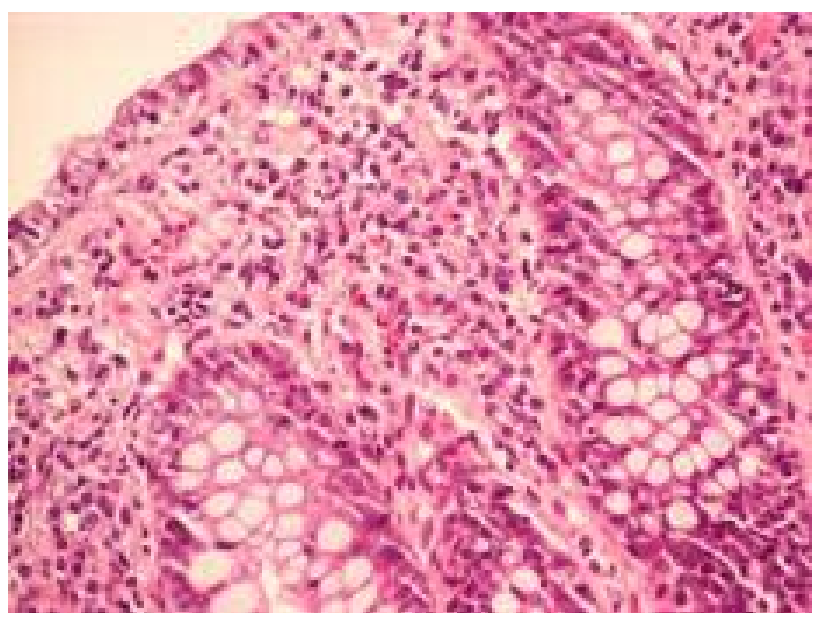

Figura 1. Mucosa cólica de 2007 com ligeiro reforço da membrana basal.

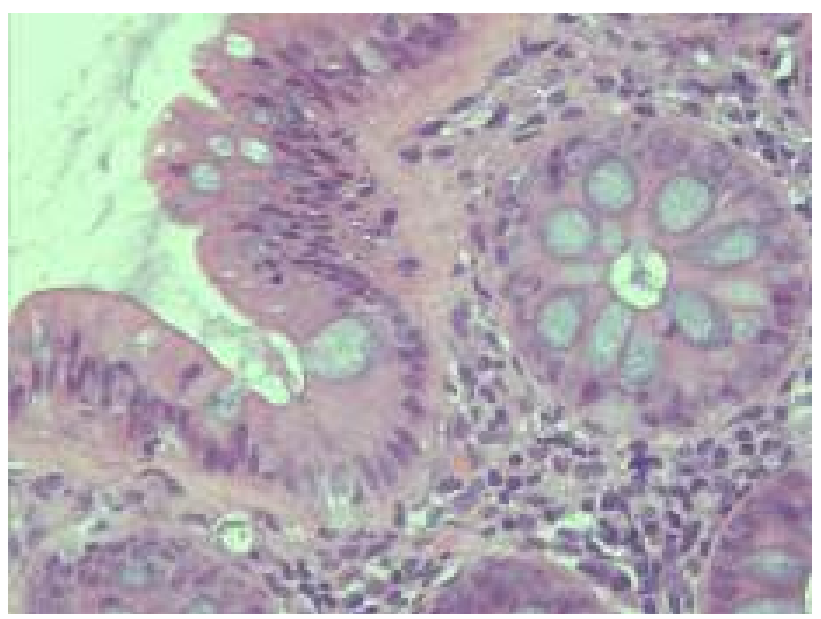

Figura 2. Mucosa cólica de 2011 com preservação da arquitectura glandular. Banda fibrosa sub-epitelial espessada.

rísticas, com dois meses de evolução, acompanhada de anorexia e perda ponderal significativa. Ao exame objectivo encontrava-se francamente desidratado e hipotenso $(60 / 49 \mathrm{mmHg})$, com abdómen mole, depressível e indolor, mas ruídos hidro-aéreos aumentados.

Analiticamente com proteína C-reactiva aumentada $(78,8 \mathrm{mg} / \mathrm{L})$ e disfunção renal aguda (ureia $176 \mathrm{mg} / \mathrm{dL}$ e creatinina $6,3 \mathrm{mg} / \mathrm{dL}$ ). Pesquisa de leucócitos nas fezes positiva. Por suspeita de enterocolite aguda cumpriu oito dias de ciprofloxacina com evolução clínica e analítica favoráveis, embora, à data da alta, ainda com dois episódios de dejecções líquidas/dia.
Repetiu estudo analítico alargado com serologias, marcadores de auto-imunidade, coproculturas e parasitológico de fezes, sendo que todos estes exames voltaram a revelar-se normais.

Realizou EDA que foi sobreponível à anterior e a ileocolonoscopia foi macroscopicamente normal mas o exame histológico (Figura 2) revelou-se a favor de colite colagenosa: preservação da arquitectura glandular com metaplasia de células de Paneth e aumento ligeiro de células inflamatórias na lâmina própria com predomínio de plasmócitos (com alguns corpos de Russell e Mott) e fibrose subepitelial com espessamento significativo focal. Iniciou tratamento com loperamida, $4 \mathrm{mg} / \mathrm{dia}$, mas com resposta apenas parcial, pelo que se associaram enemas de budesonido (ciclos mensais de sete dias) com melhoria significativa das queixas em três meses.

\section{DISCUSSÃO}

Apresentamos o caso de um doente com clínica de diarreia crónica aquosa, com exames laboratoriais e imagiológicos inicialmente normais ou inconclusivos em que, após quadro de choque com insuficiência renal aguda grave, se conseguiu estabelecer um diagnóstico preciso (com recurso a nova biópsia de cólon) e instituir terapêutica adequada, que deixou o doente assintomático.

É provável que a enterocolite tenha sido favorecida pelo quadro de diarreia crónica, já qua a descamação celular a nível intestinal favorece a translocação bacteriana, pelo que o quadro apresentado não se mostrou tão benigno como habitualmente é descrito.

A evolução constatada após corticoterapia é a usualmente observada, estando o doente assintomático desde há três anos.

Sugere-se, pois, a realização de biópsias da mucosa intestinal em casos semelhantes, dado poder haver um benefício clínico considerável com melhoria da qualidade de vida e eventualmente do prognóstico vital.

\section{REFERÊNCIAS BIBLIOGRÁFICAS}

1. Yen EF, Pardi DS. Review of the microscopic colitides. Curr Gastroenterol Rep. 2011;13(5):458-64.

2. Pardi DS, Loftus EV Jr, Smyrk TC, Kammer PP, Tremaine WJ, Schleck CD, et al. The epidemiology of microscopic colitis: a population based study in Olmsted County, Minnesota. Gut. 2007;56(4):504-8.

3. Tagkalidis P, Bhathal P, Gibson P. Microscopic colitis. J Gastroenterol He- 
patol. 2002;17(3):236-48.

4. Lindström CG. 'Collagenous colitis' with watery diarrhea: a new entity? Pathol Eur. 1976;11(1):87-9.

5. Williams JJ, Beck PL, Andrews CN, Hogan DB, Storr MA. Microscopic colitis: a common cause of diarrhoea in older adults. Age Ageing. 2010;39(2):162-8.

6. Fernández-Bañares F, Esteve M, Espinós JC, Rosinach M, Forné M, Salas A, et al. Drug consumption and the risk of microscopic colitis. Am J Gastroenterol. 2007;102(2):324-30.

7. Mäkinen M, Niemelä S, Lehtola J, Karttunen TJ. Collagenous colitis and Yersinia enterocolitica infection. Dig Dis Sci. 1998;43(6):1341-6.

8. Perk G, Ackerman Z, Cohen P, Eliakim R. Lymphocytic colitis: a clue to an infectious trigger. Scand J Gastroenterol. 1999;34(1):110-2.

9. Erim T, Alazmi WM, O'Loughlin CJ, Barkin JS. Collagenous colitis associated with Clostridium difficile: a cause effect? Dig Dis Sci. 2003;48 (7):1374-5.

10. Pinheiro I. Colite colagenosa: um diagnóstico a considerar [Collagenous colitis: a diagnosis to remember]. Med Int. 2003;10(2):83-6. Portuguese

11. Roth B, Gustafsson RJ, Ohlsson B. Auto-antibodies and their association with clinical findings in women diagnosed with microscopic colitis. PLoS ONE. 2013;8(6):e66088.

12. Madisch A, Heymer P, Voss C, Wigginghaus B, Bästlein E, Bayerdörffer
$E$, et al. Oral budesonide therapy improves quality of life in patients with collagenous colitis. Int J Colorectal Dis. 2005;20(4):312-6.

13. Guslandi M. Microscopic colitis: a therapeutic challenge. World J Gastroenterol. 2013;19(23):3531-3.

14. Ung KA, Gillberg R, Kilander A, Abrahamsson $\mathrm{H}$. Role of bile acids and bile acid binding agents in patients with collagenous colitis. Gut. 2000;46(2):170-5.

15. Miquel Plaza J, López SanRomán A, del Pozo D, Peña E, Bermejo F, Baleriola I, et al. Curso evolutivo y respuesta al tratamiento de las colitis microscópicas [Evolution and treatment response in microscopic colitis]. Gastroenterol Hepatol. 2001;24(9):433-9. Spanish

\section{CONFLITO DE INTERESSES}

Os autores declaram não ter conflitos de interesses.

\section{ENDEREÇO PARA CORRESPONDÊNCIA}

Sandra A. Morais

Rua Dr. Eduardo Torres, 4464-513 Senhora da Hora

E-mail: sandramorais13@hotmail.com

Recebido em 18-05-2015

Aceite para publicação em 30-11-2015

\begin{abstract}
COLLAGENOUS COLITIS: AN UNDERDIAGNOSED CAUSE OF CHRONIC DIARRHEA

Microscopic colitis is a disorder that includes collagenous colitis and lymphocytic colitis. It is considered to be a common cause of chronic diarrhea. Although it is benign, it can have a significant impact on the patient's quality of life. The diagnosis is exclusively histological. Although microscopic colitis was first described in 1976, it has only recently been recognized as a common cause of diarrhea. Many clinicians are not yet aware of this entity. We present the case a patient with chronic watery diarrhea, initially without significant systemic repercussions and with normal or inconclusive laboratory or imaging tests. This evolved into hypovolemic shock with severe renal dysfunction. Greater awareness of this condition by clinicians, leading to the performance of timely biopsies, required for the correct diagnosis and appropriate therapy, is required.
\end{abstract}

Keywords: Collagenous Colitis; Microscopic Colitis; Chronic Diarrhea. 